\title{
Evaluación de metales redox-sensitivos como proxies de paleoxigenación en un ambiente marino hipóxico del norte de Chile
}

\author{
Redox-sensitive metals evaluation as proxis of paleoxygenation in a hypoxic marine \\ environment of northern Chile
}

\begin{abstract}
JORGE VALDÉS ${ }^{1,2}$
${ }^{1}$ Instituto de Investigaciones Oceanológicas, Facultad de Recursos del Mar, Universidad de Antofagasta, Casilla 170, Antofagasta, Chile; e-mail: jvaldes@uantof.cl

${ }^{2}$ Institut de Recherche pour le Développement, UR 055 PALEOTROPIQUE, Centre de Recherche Ile de France, 32 Avenue Henri Varagnat, F-93143 Bondy Cedex, France
\end{abstract}

\begin{abstract}
RESUMEN
Se analizó la distribución de molibdeno, cadmio, zinc, vanadio y níquel, todos metales redox-sensitivos, bajo una perspectiva espacial (sedimento superficial) y temporal (testigos de sedimento), con el propósito de determinar su potencial como indicadores de paleoxigenación en la bahía Mejillones del Sur, un ambiente hipóxico del norte de Chile. El aporte litogénico (de acuerdo a aluminio) fue descartado. Además, el flujo biogénico no es el único factor que controla la preservación de metales en esta bahía. La variación espacial en la concentración de metales fue investigada en relación con la oxigenación de las aguas de fondo. Los resultados mostraron que solo el $\mathrm{Ni}$ y el Cd presentan una correlación significativa con el oxígeno disuelto, aumentando sus concentraciones hacia el sector más profundo de la bahía, en donde prevalecen condiciones de mínimo oxígeno, con valores cercanos a $0,02 \mathrm{~mL} \mathrm{~L}^{-1}$. La variación temporal en la concentración de metales redox-sensitivos, registrada en tres testigos de sedimento que cubren los pasados 2.000 años, mostró que, en general, la oxigenación de fondo de la bahía ha cambiado desde un ambiente más oxigenado en el pasado, hacia un ambiente menos oxigenado en el presente. Se propone que el flujo biogénico a través de una oxiclina bien desarrollada, asociado a una condición anóxica de la interfase agua-sedimento son los principales factores que permiten explicar la concentración de metales que se preserva en los sedimentos marinos de Mejillones durante condiciones no-El Niño/La Niña. Cuando El Niño está presente en la bahía, se profundiza la oxiclina, aumentando el nivel de oxigenación de las aguas subsuperficiales al interior de la bahía, lo que provoca que la precipitación de metales hacia los sedimentos disminuya, favoreciendo, a su vez, la liberación de metales desde la interfase agua-sedimento. Basado en esto, se plantea que las diferencias en la concentración de metales registrada entre las muestras de la parte superior de los testigos (correspondientes a antes de 1996) y las muestras superficiales (correspondientes al año 2000) y tomadas en la misma zona de los testigos, pueden ser atribuidas al efecto de El Niño 1997-1998, el cual provocó un brusco descenso en el flujo de metales (principalmente $\mathrm{Ni}$ y $\mathrm{Cd}$ ) hacia los sedimentos, debido a una condición más oxigenada de la columna de agua. Este trabajo muestra que de los cinco metales analizados, solo en $\mathrm{Ni}$ y el $\mathrm{Cd}$ parecen tener un potencial como indicadores de paleoxigenación en la bahía Mejillones. Sin embargo, su uso está restringido para identificar cambios extremos desde una condición anóxica a una óxica en los sedimentos de fondo de esta bahía.
\end{abstract}

Palabras clave: metales redox-sensitivos, paleoxigenación, sedimentos marinos, Zona de Mínimo Oxígeno, bahía Mejillones.

\begin{abstract}
The distribution of molybdenum, cadmium, zinc, vanadium and nickel, all redox-sensitive metals, was analyzed under a spatial (surface sediment samples) and temporal (core samples) perspective, in order to identify the potential of these metals for paleoxygenation interpretations in Mejillones bay, a hypoxic environment of northern Chile. Lithogenic supply (according to aluminum) was discarded. Biogenic flux is not the unique factor that controls the mechanism of metals preservation in sediments of this bay. Spatial variation of metals concentration was investigated in relation to the bottom water oxygenation in each sampling station. Results showed that only $\mathrm{Ni}$ and $\mathrm{Cd}$ present a strong correlation with dissolved oxygen, increasing their concentration toward the deepest area of the bay were an oxygen minimum zone prevails with values around $0.02 \mathrm{~mL} \mathrm{~L}^{-1}$. The temporal variation in redox-sensitive metals, recorded in three sediments core covering the last 2,000 years, showed that bottom oxygenation of the bay changed from a more oxygenated environment in the past to a less oxygenated environment at present. A biogenic flux across a very well
\end{abstract}


developed oxycline, associated to an anoxic sediment-water interface, are proposed to be the principal factors to explain the metal concentrations preserved in Mejillones sediments during non-El Niño condition. When El Niño is present in the bay, the water column turn oxic, and the precipitation of redox-sensitive metals drops; so the concentration of metals decrease in the sediments. Thus, differences in concentration of metals in core top (sampled in 1996) and surface sediments (sampled in 2000) in the same area of the bay, were attributed to El Niño 1997-1998 that provoked a decrease in the flux of metals (principally nickel and cadmium) toward the sediments in a more oxygenated bottom environment. All these information show that among the five metals analyzed, only nickel and cadmium seem to record paleoxygenation conditions in Mejillones. However their use as proxy is limited to the cases when extreme changes from anoxic to oxic conditions occurred in bottom sediments of this bay.

Key words: redox-sensitive metals, paleoxygenation, marine sediments, Oxygen Minimum Zone, Mejillones bay.

\section{INTRODUCCIÓN}

Prácticamente todos los metales son encontrados en el océano, en forma disuelta o particulada, siendo introducidos por ríos, fuentes hidrotermales, difusión desde los sedimentos, vientos, y actividad antrópica. Muchos de estos metales reaccionan a la condición de oxigenación de su entorno, por lo que son llamados redox-sensitivos, y el estudio de su comportamiento diagenético frente a estos cambios de la oxigenación provee las bases para interpretar variaciones en el estado redox de los sedimentos marinos (Didyk et al. 1978, Malcolm 1985, van Geen et al. 1995, Brown et al. 2000).

El registro sedimentario de muchos metales redox-sensitivos aporta importante información respecto del estado de oxigenación imperante en el ambiente depositacional cuando dicho sedimento fue depositado. Este comportamiento particular ha permitido su uso en reconstrucciones sobre la oxigenación de aguas de fondo en el pasado, basado en variaciones de la concentración a lo largo de secuencias sedimentarias marinas (Pedersen et al. 1988, Calvert \& Pedersen 1993, Dean 1993, Jones \& Manning 1994, Rosenthal et al. 1995, Calvert et al. 1996, Dean et al. 1997). En estudios paleoceanográficos, la evolución de la oxigenación subsuperficial constituye una información importante para comprender diferentes procesos oceanológicos como la productividad primaria, la surgencia y la dinámica de las masas de agua (Didyk et al. 1978, Anderson \& Gardner 1989, Dean 1993, Kaiho 1994, Legeleux et al. 1994).

Los elementos traza que responden a variaciones de la condición de oxigenación del ambiente de depositación marino generalmente se pueden agrupar en dos categorías. La primera corresponde a aquellos elementos cuya valencia varía en función del potencial redox. En esta categoría están el manganeso $(\mathrm{Mn})$, el cual forma un óxido insoluble en un ambiente oxigenado, y el molibdeno (Mo), renio (Re), uranio (U), y el vanadio $(\mathrm{V})$, los cuales forman especies anióni- cas altamente solubles en ambientes oxigenados, pero que son reducidos a especies reactivas insolubles de menor valencia bajo condiciones anóxicas. La segunda categoría agrupa a aquellos elementos cuya valencia no cambia, pero que bajo condiciones anóxicas forman sulfuros insolubles. Entre estos están el cadmio (Cd), cobre $(\mathrm{Cu})$, níquel $(\mathrm{Ni})$ y zinc $(\mathrm{Zn})$, los cuales usualmente son removidos de la solución en presencia de $\mathrm{H}_{2} \mathrm{~S}$ (Calvert \& Pedersen 1993). El comportamiento geoquímico de los metales redox-sensitivos más comúnmente utilizados en reconstrucciones paleoceanográficas es ampliamente discutido en Calvert \& Pedersen (1993), Helz et al. (1996), Morford \& Emerson (1999), Bostick et al. (2000), Brown et al. (2000), Crusius \& Thomson (2000), Hammond et al. (2000), Zheng et al. (2000), Morford et al. (2001) y Russell \& Morford (2001)

El uso de estos metales para reconstruir características pasadas de los ambientes marinos de depositación requiere de una etapa de calibración, debido a que el comportamiento geoquímico y su potencial como proxy de paleoxigenación puede variar de un lugar a otro.

\section{Un ambiente costero hipóxico en el norte de Chile}

Mejillones es una pequeña bahía situada en el Trópico de Capricornio ( $\left.23^{\circ} \mathrm{S}\right)$, a orillas del desierto de Atacama. Rodríguez et al. (1991) demostraron, por medio de análisis de nutriente e imágenes satelitales de la Temperatura Superficial del Mar (TSM), que esta bahía está localizada cerca de un centro de surgencia. Este centro, uno de los más productivos del norte de Chile, es conocido como Punta Angamos $\left(23^{\circ} \mathrm{S}\right)$, y ha sido objeto de numerosos estudios en los últimos años (Escribano 1998, González et al. 1998, 2000, Marín \& Olivares 1999, Escribano \& Hidalgo 2000, Sobarzo \& Figueroa 2001, Thomas et al. 2001). La bahía Mejillones del Sur, que forma parte de este sistema, actúa como un centro de depositación 
de la abundante productividad biológica de la zona. Mediciones de productividad primaria en esta bahía han arrojado valores de $138 \mathrm{mg}$ $\mathrm{C} \mathrm{m}^{-3} \mathrm{~h}^{-1}$ (Rodríguez et al. 1986), mientras que Marín et al. (1993) registraron una productividad anual de $1.070 \mathrm{~g} \mathrm{C} \mathrm{m}^{2}$, resaltando que esta alta productividad del interior de la bahía era controlada por los eventos de surgencia que ocurren en Punta Angamos. Recientemente Marín \& Olivares (1999) propusieron un modelo conceptual para explicar la alta productividad biológica de la bahía Mejillones. En este modelo la surgencia se localiza fuera de la bahía (en Punta Angamos), y las aguas enriquecidas con nutrientes ingresan a ella por superficie, amplificando el efecto fertilizador debido a una mayor estabilidad termal de la columna de agua. Esta situación es favorecida por la configuración geográfica de la bahía, en relación a los vientos predominantes.

Una de las características oceanológicas que explica el potencial paloecanográfico de los sedimentos acumulados en la bahía Mejillones es la pobreza de oxígeno de su ambiente depositacional. Perfiles de oxígeno disuelto medidos en un ciclo anual muestran que a profundidades mayores a los $50 \mathrm{~m}$, la bahía está bajo los efectos de la Zona de Mínimo Oxígeno (ZMO) (Navea \& Miranda 1980, Escribano 1998, Valdés 1998), lo cual restringe fuertemente la presencia de macrofauna bentónica (Zúñiga 1974, Zúñiga et al. 1983). Estos factores han favorecido la acumulación y posterior preservación de sedimentos diatomaceos laminados, ricos en materia orgánica, y con mínimas señales de bioturbación, conformando un importante registro sobre las condiciones paleo-océano-climáticas imperantes en la zona, durante el pasado reciente (Ortlieb et al. 1994, 2000, Valdés 1998, Vargas 1998, 2002, Valdés et al. 2000, 2003, Valdés \& Ortlieb 2001, Páez et al. 2001).

El conocimiento sobre la variabilidad temporal de estos ambientes pobremente ventilados, en términos de su intensidad y duración, pueden ayudar a entender procesos tan importantes como la productividad biológica de las aguas superficiales, el ciclo del carbono y la preservación de materia orgánica en los sedimentos de fondo. Sin embargo, toda interpretación paleoambiental requiere de una selección de los estimadores adecuados al proceso y/o mecanismo oceanológico que se pretende estudiar. Por ello, el presente trabajo tiene como objetivo estudiar el potencial como estimadores de paleoxigenación, de un conjunto de metales redox-sensitivos registrados en los sedimentos de la bahía Mejillones del Sur.

\section{MATERIALES Y MÉTODOS}

\section{Campañas de muestreo}

Se realizaron dos campañas de muestreo a bordo de la embarcación oceanográfica Purihaalar de la Universidad de Antofagasta. En la primera campaña, realizada en junio de 1996, se recuperaron tres columnas de sedimento marino con un sacatestigos de gravedad tipo Plheger, en la zona media de la bahía Mejillones (Fig. 1). El testigo $32 \mathrm{~A}$ fue seccionado en submuestras de $4 \mathrm{~cm}$, el testigo $32 \mathrm{E}$ fue seccionado en submuestras de $2 \mathrm{~cm}$ y el testigo $32 \mathrm{G}$ fue seccionado en submuestras de $1 \mathrm{~cm}$, de acuerdo a criterios estratigráficos observados al momento de abrir los testigos.

Posteriormente, en junio del año 2000, se recuperaron 36 muestras de sedimento superficial $(0,5 \mathrm{~cm})$, con un mini box-core de 225 $\mathrm{cm}^{2}$ (Fig. 1). Las muestras fueron removidas desde el centro del box-core, con una espátula de plástico, para minimizar la contaminación por metales. Conjuntamente se midió el contenido de oxígeno disuelto de las aguas subsuperficiales (a $5 \mathrm{~m}$ del fondo), con un correntómetro Aandera RCM 9, equipado con un sensor de oxígeno.

Todas las muestras de sedimento fueron colocadas en bolsas de plástico y preservadas a $4^{\circ}$ $\mathrm{C}$ hasta comenzar su tratamiento. Posteriormente las muestras fueron puestas a secar a $40^{\circ} \mathrm{C}$ hasta la perdida total de agua, y maceradas en un mortero de ágata.

\section{Procedimiento analítico}

El análisis de metales se realizó con un espectrofotómetro de absorción atómica de llama marca Perkin Elmer modelo 1100 B, según el procedimiento descrito por el Método Estándar 3050 de la EPA. Durante la rutina de análisis algunas muestras fueron seleccionadas para realizar adiciones estándar con el propósito de identificar efectos de matriz, lo cual fue descartado. También se realizaron análisis de blancos, duplicados y material de referencia PACS-1 K1A OR6 y MESS-1 K1A OR9 de la National Research Council, Canadá. Se determinó un error de un $5 \%$. En el sedimento superficial y en el testigo 32A se midió la concentración de molibdeno (Mo), cadmio ( $\mathrm{Cd})$, zinc ( $\mathrm{Zn})$, vanadio (V) y níquel (Ni). En el testigo $32 \mathrm{G}$ se midió la concentración de Mo y $\mathrm{Cd}$, y en el testigo $32 \mathrm{E}$ se midió la concentración de $\mathrm{Cd}$. Además se midió la concentración de aluminio (Al) en las muestras de sedimento superficial para evaluar el aporte de material continental. 


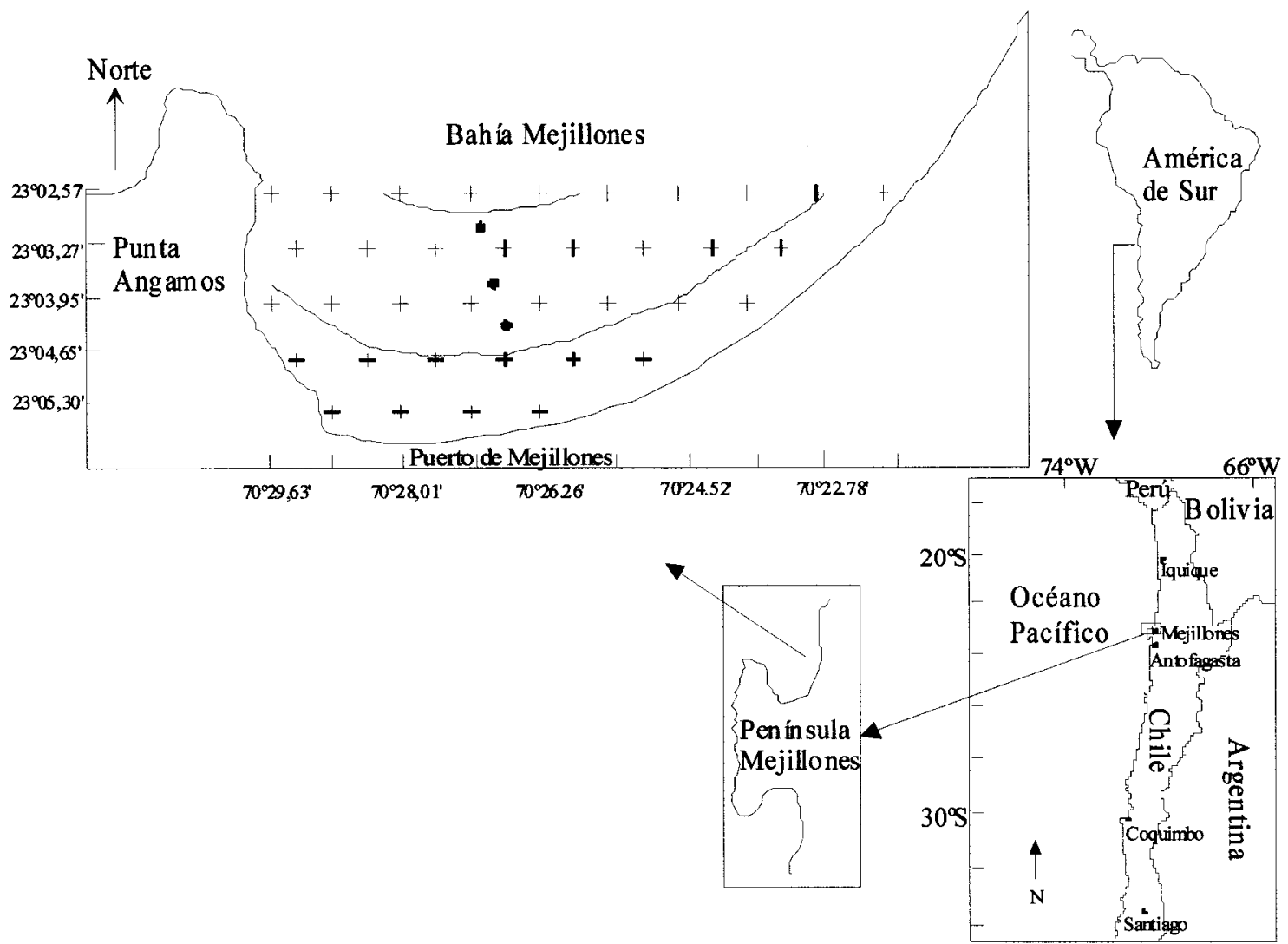

Fig. 1: Ubicación de la bahía Mejillones en la costa norte de Chile. Las cruces corresponden a las muestras de sedimento superficial y los círculos corresponden a los testigos 32A, 32E y 32G, de norte a sur, respectivamente.

Location map of Mejillones bay on the northern coastal off Chile. Crosses corresponding to surface samples and fill circles correspond to core samples $32 \mathrm{~A}, 32 \mathrm{E}$ and $32 \mathrm{G}$, from north to south, respectively.

La campaña del año 2000 permitió construir mapas de distribución espacial en el programa SURFER 8.0, sobre la base del método de interpolación de Shepard (Renka 1988).

\section{RESULTADOS}

\section{Oxigenación subsuperficial}

El contenido de oxígeno disuelto del fondo de la bahía de los tres meses anteriores al muestreo del año 2000 fueron promediados para construir un mapa de distribución espacial de oxigenación subsuperficial (Fig. 2). Basados en la escala de oxigenación propuesta por Bernhard \& Sen Gupta (1999) se identificaron las zonas óxica, disóxica, y micróxica, al igual que la Zona de Mínimo Oxígeno $\left(<0,5 \mathrm{~mL} \mathrm{~L}^{-1}\right)$. Entre los 10 y $100 \mathrm{~m}$ de profundidad, el oxíge- no disuelto varió entre $4,5 \mathrm{~mL} \mathrm{~L}^{-1}$ en la zona costera y $0,01 \mathrm{~mL} \mathrm{~L}^{-1}$ en la zona más profunda. De acuerdo a esta distribución, la mayor parte de la zona estudiada está bajo una condición de mínimo oxígeno $\left(<0,1 \mathrm{~mL} \mathrm{~L}^{-1}\right)$. En general, los ambientes ventilados prevalecen en la zona más cercana a la línea de costa, mientras que los ambientes pobres en oxígeno se restringen a la zona más profunda de la bahía.

\section{Molibdeno}

La concentración media de Mo fue la misma en el sedimento superficial y en los testigos $(50,5$ $\mathrm{mg} \mathrm{kg}^{-1}$ ) (Tabla 1). El sedimento superficial mostró dos zonas de alta concentración, una hacia el sur de la bahía, frente al puerto, y otra en el límite norte del área de estudio (Fig. 3). Los valores más bajos fueron registrados en la zona este de la bahía. El testigo 32G mostró dos sec- 


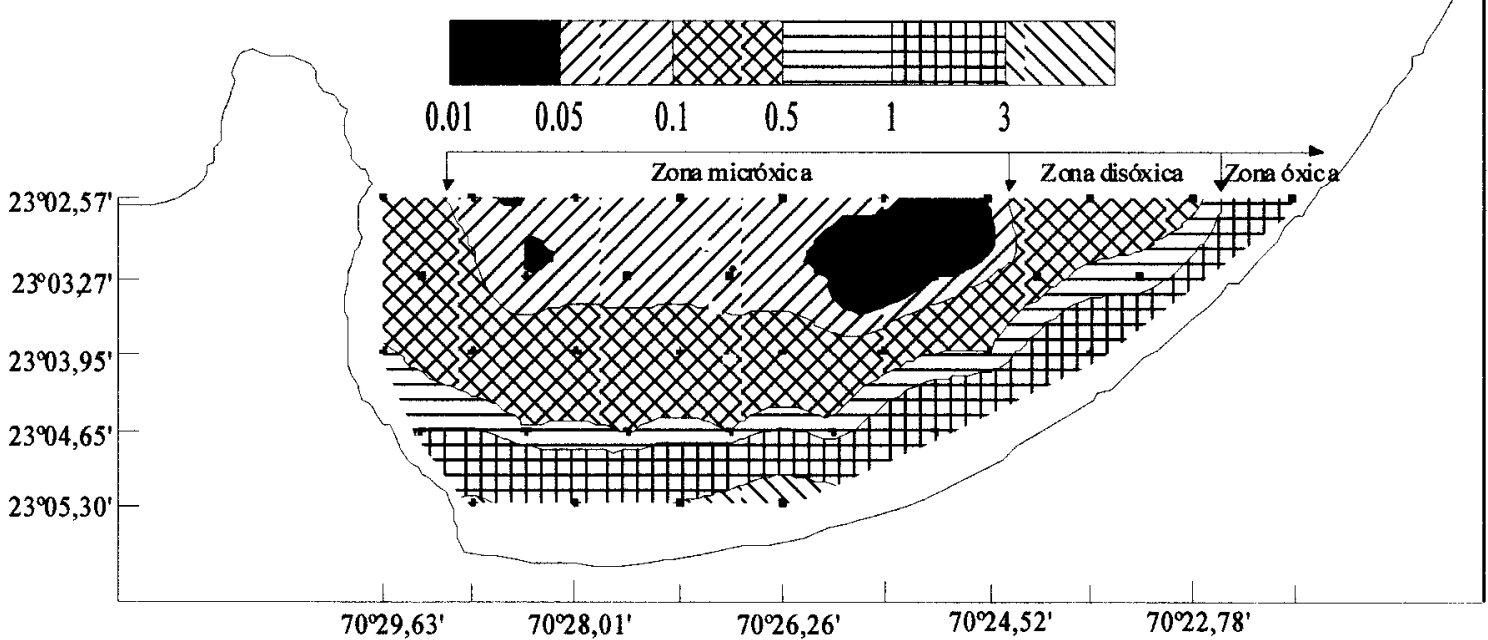

Fig. 2: Oxígeno disuelto de fondo ( $5 \mathrm{~m}$ sobre el fondo marino) para el período abril-junio del año 2000. Escala de oxigenación según Berhard \& SenGupta (1999). Se muestran los puntos de muestreo. ZMO es Zona de Mínimo Oxígeno.

Bottom dissolved oxygen (5 m over sea floor) for period April-June. Oxygenation scale according Bernhard \& SenGupta (1999). Sampling points are showed. OMZ is Oxygen Minimum Zone.

TABLA 1

Estadística descriptiva de las mediciones de concentración de metales en muestras de sedimento superficial y testigos; (n) es número de muestras; concentración en $\mathrm{mg} \mathrm{kg}^{-1}$

Descriptive statistic for the metal concentrations measurements from surface and core sediment samples; $\mathrm{n}$ is the number of samples; concentration in $\mathrm{mg} \mathrm{kg}^{-1}$

\begin{tabular}{|c|c|c|c|c|c|c|}
\hline Tipo de muestra & Metal & $\mathrm{n}$ & Media & $\mathrm{DE}$ & Valor mínimo & Valor máximo \\
\hline \multirow{6}{*}{$\begin{array}{l}\text { Muestras } \\
\text { superficiales }\end{array}$} & Mo & 35 & 50,5 & 28,2 & 10,0 & 118,0 \\
\hline & $\mathrm{Cd}$ & 35 & 21,9 & 11,1 & 3,0 & 38,0 \\
\hline & $\mathrm{Zn}$ & 35 & 29,8 & 12,5 & 10,0 & 62,0 \\
\hline & $\mathrm{V}$ & 35 & 43,9 & 26,5 & 1,0 & 97,0 \\
\hline & $\mathrm{Ni}$ & 35 & 20,6 & 10,4 & 2,0 & 36,0 \\
\hline & $\mathrm{Al}$ & 35 & 3378,1 & 859,1 & 715,0 & 4910,0 \\
\hline \multirow{5}{*}{$\begin{array}{l}\text { Testigos de } \\
\text { sedimento }\end{array}$} & Mo & 70 & 50,5 & 8,9 & 25,8 & 76,3 \\
\hline & $\mathrm{Cd}$ & 106 & 37,3 & 10,4 & 19,0 & 60,1 \\
\hline & $\mathrm{Zn}$ & 11 & 16,6 & 6,1 & 10,7 & 26,9 \\
\hline & $\mathrm{V}$ & 11 & 134,4 & 12,1 & 120,2 & 154,5 \\
\hline & $\mathrm{Ni}$ & 11 & 40,0 & 13,6 & 18,8 & 72,7 \\
\hline
\end{tabular}

tores de bajo contenido de Mo, uno entre 45 y $41 \mathrm{~cm}$, y otro entre 26 y $22 \mathrm{~cm}$ de profundidad (Fig. 3). El testigo 32A mostró un aumento de la concentración hacia el sedimento superficial con dos fuertes caídas a los 30 y $15 \mathrm{~cm}$. Ambos testigos mostraron un incremento de la concentración hacia el sedimento superficial. La concentración de Mo en la muestra superficial de los testigos (sedimento reciente) fue menor que la registrada, en el mismo sector, en la campaña del año 2000.

\section{Cadmio}

La concentración media de $\mathrm{Cd}$ en el sedimento superficial fue de $21,9 \mathrm{mg} \mathrm{kg}^{-1}$, mientras que en 


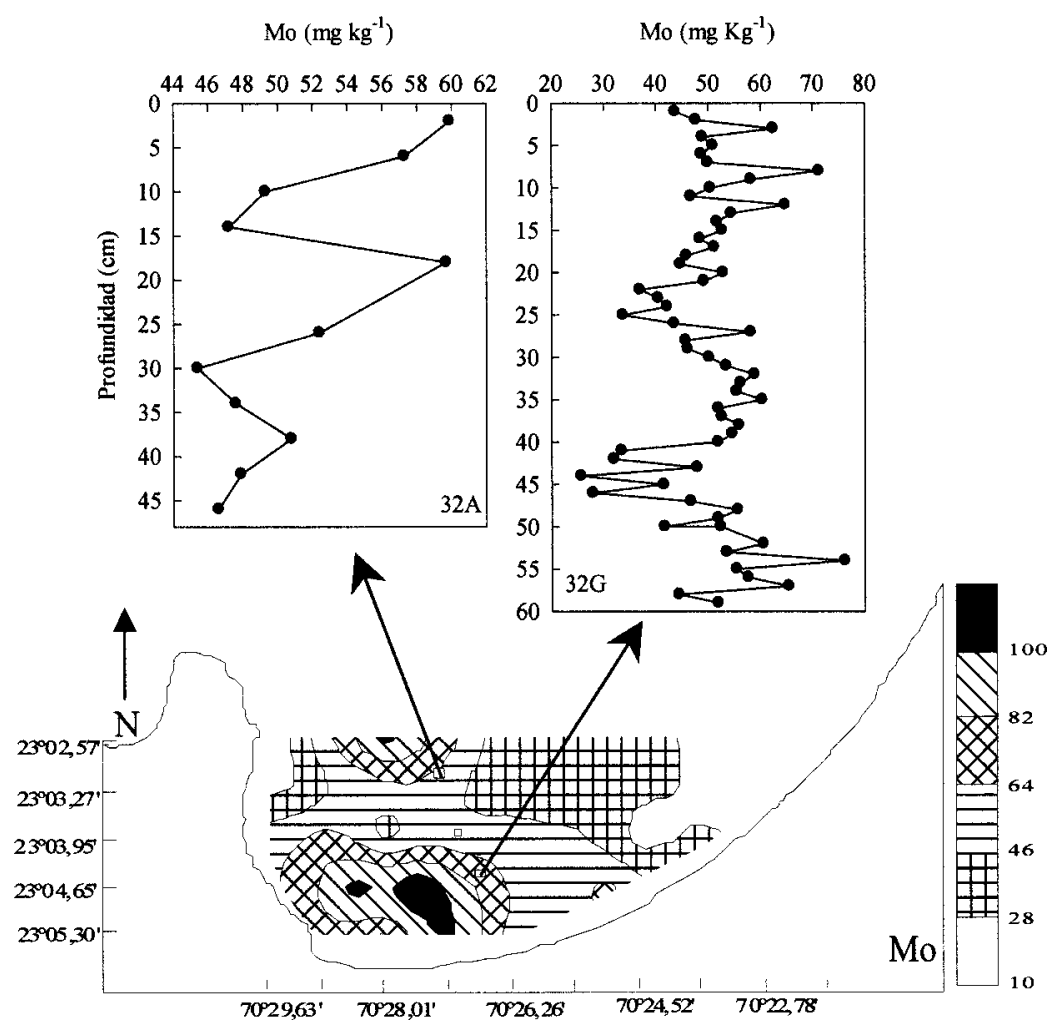

Fig. 3: Distribución espacial de Mo en sedimentos superficiales, y perfiles en dos testigos. Se indica la ubicación de colecta de ambos testigos.

Contour map of Mo concentration in surface samples, and profile in two sediment cores. Sampling station of both cores are indicated.

los testigos fue de $37,3 \mathrm{mg} \mathrm{kg}^{-1}$ (Tabla 1). Desde una perspectiva espacial, los valores más bajos se registraron en la zona costera, principalmente en el sector sureste de la bahía, mientras que se registró un notorio aumento hacia la zona profunda (Fig. 4). Todos los testigos mostraron un aumento de la concentración de $\mathrm{Cd}$ hacia el sedimento superficial. Los tres testigos mostraron una sección de alta concentración de Cd entre los 42 y $26 \mathrm{~cm}$ de profundidad, mientras que la concentración de la muestra superficial (sedimento reciente) en los tres casos fue mas alta que la registrada, en los mismos puntos de muestreo, en la campaña del año 2000.

\section{Zinc}

La concentración media de las muestras de sedimento superficial fue más alta que la media registrada en los testigos (Tabla 1). El mapa de distribución espacial mostró una zona de baja concentración en la zona costera y en el noroeste de la bahía, separadas por una banda de alta concentración (Fig. 5). En el caso del testigo se registró un incremento de la concentración hacia el sedimento superficial (Fig. 5). La concentración registrada en la muestra superficial del testigo (sedimento reciente) fue mayor que la registrada, en el mismo lugar, en la campaña del año 2000.

\section{Vanadio}

La distribución espacial mostró una banda de alta concentración en el centro de la bahía (Fig. 6). En general, valores bajos se encontraron en la zona costera y altos en la zona más profunda. En el testigo se registró una sección de alto contenido de $\mathrm{V}$ entre la base y los $20 \mathrm{~cm}$ de profundidad (Fig. 6). Las concentración media de $\mathrm{V}$ fue más alta en el testigo que en las muestras de sedimento superficial (Tabla 1).

\section{Níquel}

Las muestras superficiales y las del testigo presentaron una concentración media de 20,6 $\mathrm{mg} \mathrm{kg}^{-1}$ y $40 \mathrm{mg} \mathrm{kg}^{-1}$, respectivamente (Tabla 1 


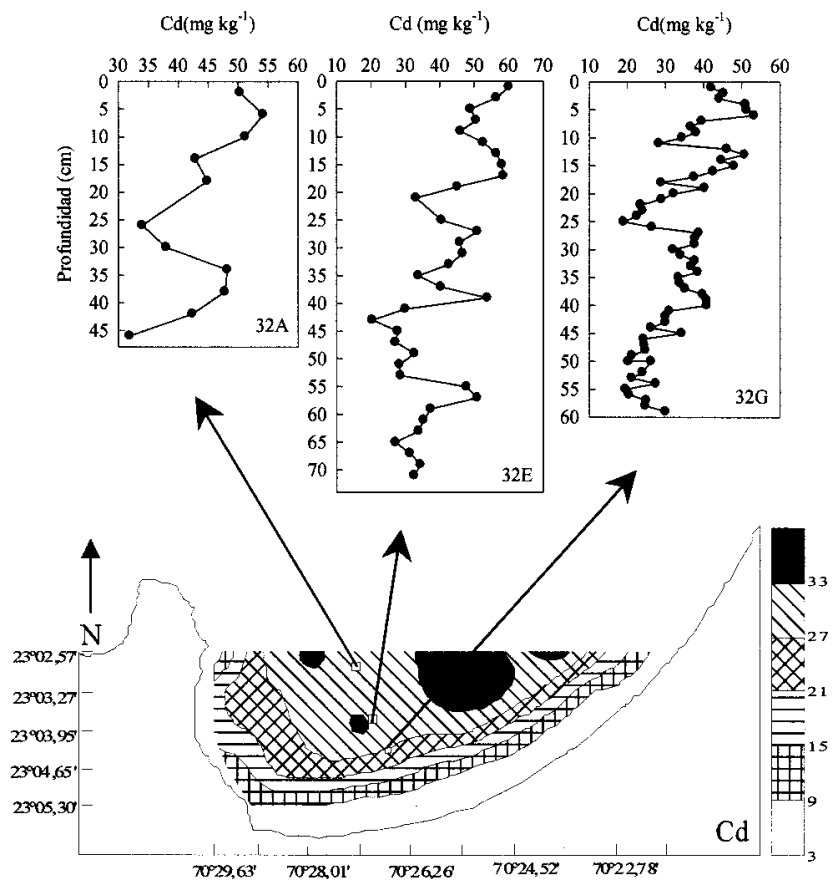

Fig. 4: Distribución espacial de Cd en sedimentos superficiales, y perfiles en tres testigos. Se indica la ubicación de colecta de los testigos.

Contour map of $\mathrm{Cd}$ concentration in surface samples, and profile in three sediment cores. Sampling station of cores are indicated.

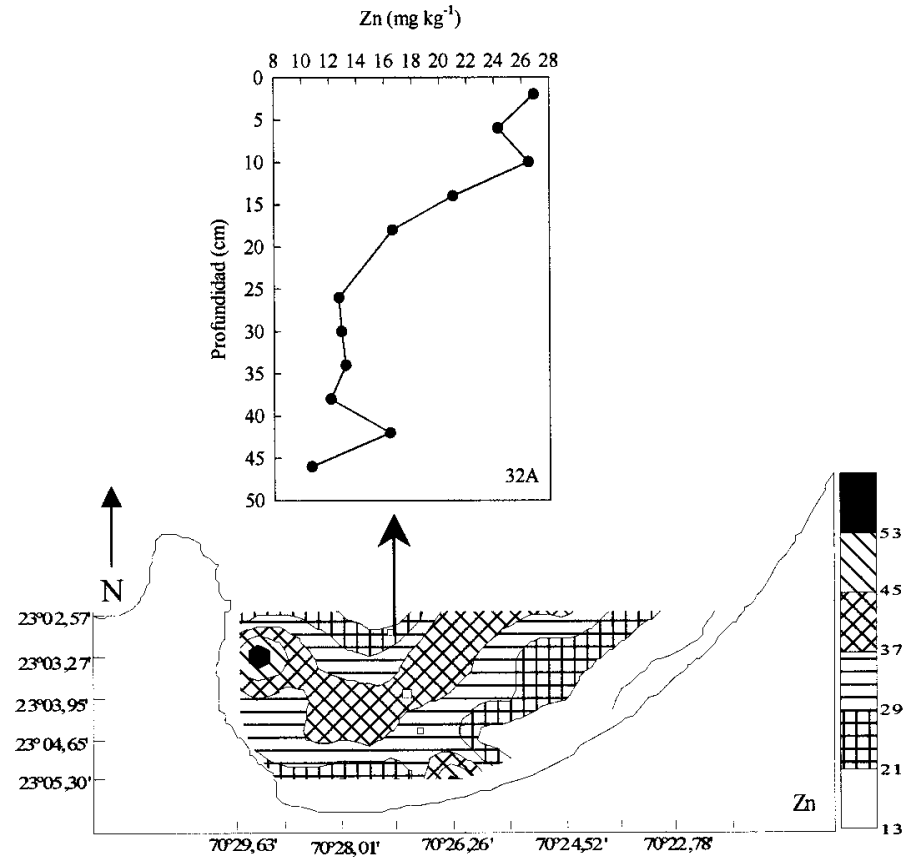

Fig. 5: Distribución espacial de Zn en sedimentos superficiales, y perfil en un testigo. Se indica la ubicación de colecta del testigo.

Contour map of $\mathrm{Zn}$ concentration in surface samples, and profile in sediment cores 32A. Sampling station of core is indicated. 


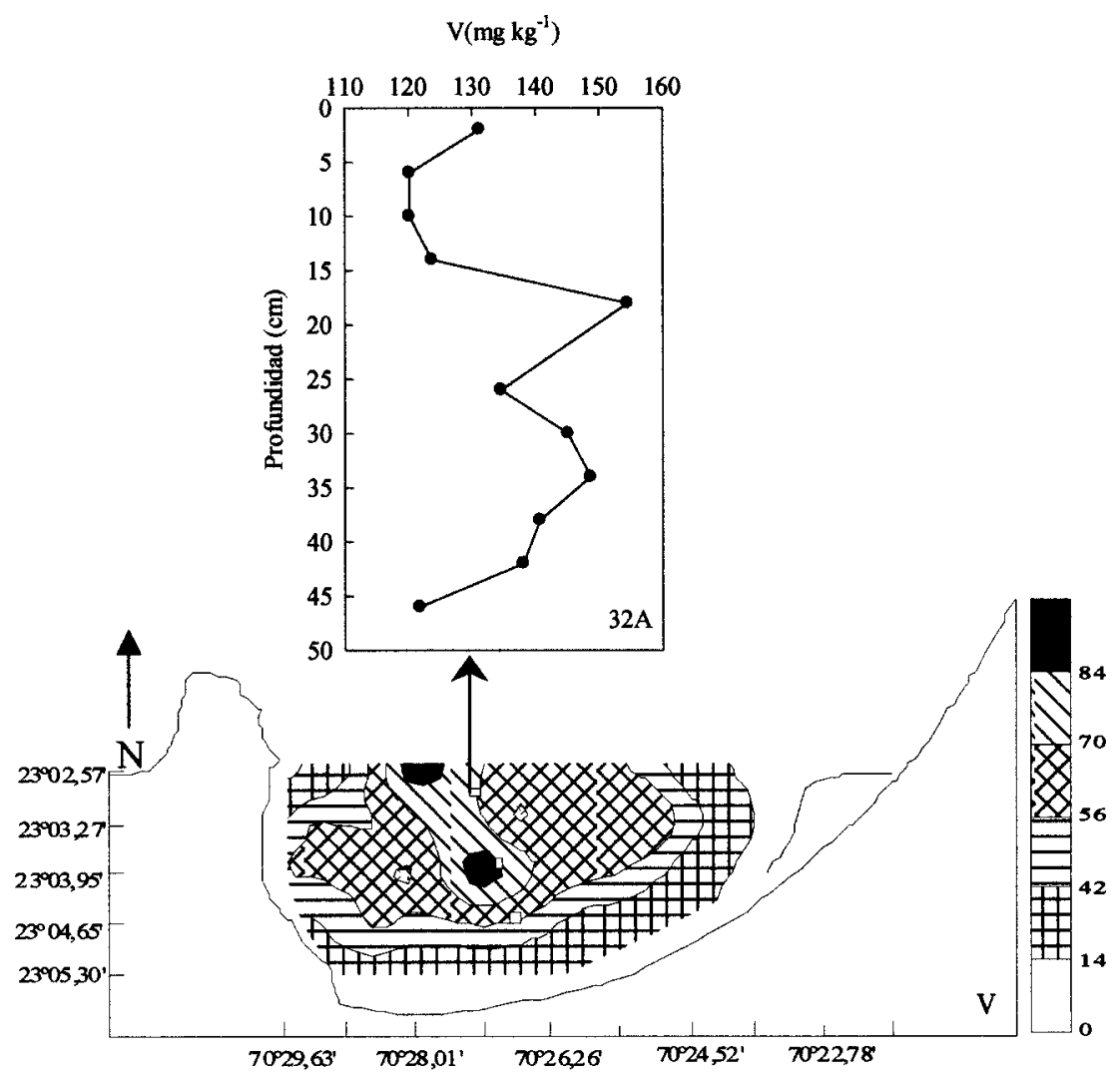

Fig. 6: Distribución espacial de V en sedimentos superficiales, y perfil en un testigo. Se indica la ubicación de colecta del testigo.

Contour map of $\mathrm{V}$ concentration in surface samples, and profile in sediment core $32 \mathrm{~A}$. Sampling station of core is indicated.

La distribución espacial de Ni mostró la misma tendencia que el $\mathrm{Cd}$, con valores bajos en la zona costera y valores altos hacia la zona más profunda (Fig. 7). El testigo mostró poca variación de la concentración entre la base y los 16 $\mathrm{cm}$ de profundidad, y una fuerte fluctuación en la parte superior (Fig. 7). La concentración medida en la muestra superficial del testigo fue mayor que la registrada, en la misma zona, en la campaña del año 2000.

El cálculo del coeficiente de correlación de Pearson entre los metales, oxígeno y la profundidad de muestreo, de la campaña del año 2000, se muestran en la Tabla 2. Se encontró una correlación significativa $(\mathrm{P}<$ $0,001)$ entre el $\mathrm{Cd}, \mathrm{Ni}$, profundidad y oxígeno disuelto. El V se correlacionó significativamente con el $\mathrm{Cd}$, Ni pero no con el oxígeno disuelto ni la profundidad. También se calculó el coeficiente de correlación para el testigo $32 \mathrm{~A}$ debido a que en él se midieron todos los metales. Los resultados (Tabla 3) muestran que el $\mathrm{Cd}$, el Ni y el $\mathrm{Zn}$ presentan una correlación significativa.

\section{DISCUSIÓN}

\section{Sedimentos superficiales}

Se observaron diferencias en las características texturales de los sedimentos de la bahía. Aquellos acumulados hacia la zona más profunda (dentro de la ZMO) correspondieron a limo de color oliva-gris, ricos en materia orgánica, mientras que en la zona más somera predominó la arena muy fina. En la zona más profunda, Valdés (1998) y Valdés et al. (2000, 2003) han registrado concentraciones de carbono orgánico total de hasta $10 \%$, cuyo origen está en la alta productividad biológica de las aguas superficiales, y en la eficiente preservación del ambiente de depositación, caracterizado por el bajo contenido de oxígeno disuelto. 

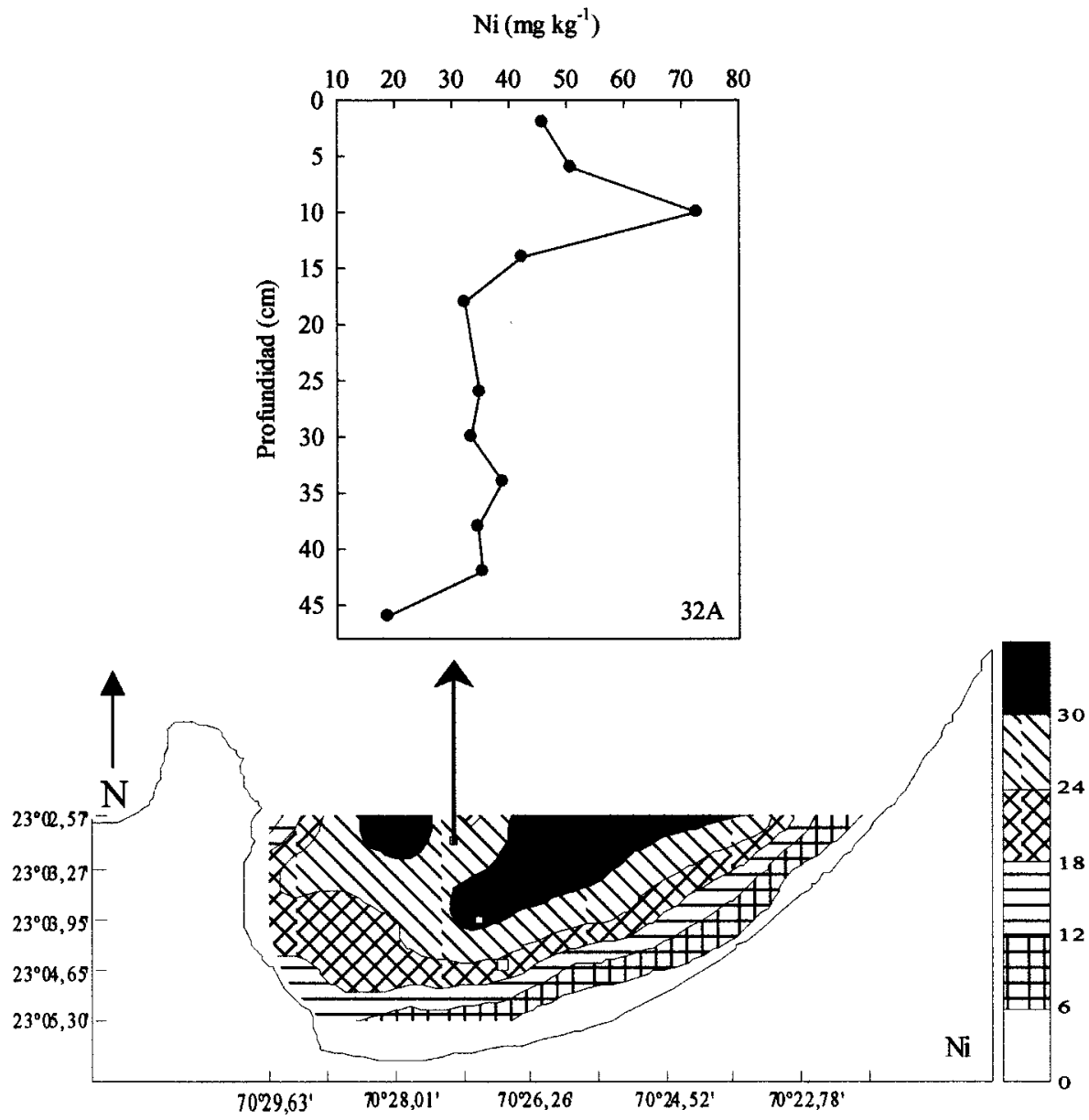

Fig. 7: Distribución espacial de Ni en sedimentos superficiales, y perfil en un testigo. Se indica la ubicación de colecta del testigo.

Countor map of $\mathrm{Ni}$ concentration in surface samples, and profile in sediment core 32A. Sampling station of core is indicated.

TABLA 2

Coeficiente de correlación de Pearson para los parámetros de muestras de sedimentos superficial.

En negritas se indican las correlaciones significativas $(\mathrm{P}<0,001)$

Pearson's correlation table for parameters measurement in surface samples from Mejillones bay.

Significant correlations are indicated in bold typing $(\mathrm{P}<0.001)$

\begin{tabular}{|c|c|c|c|c|c|c|c|}
\hline & $\mathrm{Cd}$ & $\mathrm{Zn}$ & Mo & $\mathrm{Ni}$ & V & Oxígeno & Profundidad \\
\hline $\mathrm{Zn}$ & 0,374 & & & & & & \\
\hline Mo & 0,032 & 0,074 & & & & & \\
\hline $\mathrm{Ni}$ & 0,944 & 0,483 & 0,112 & & & & \\
\hline V & 0,693 & 0,406 & 0,462 & 0,737 & & & \\
\hline Oxígeno & $-0,761$ & $-0,134$ & 0,123 & $-0,727$ & $-0,475$ & & \\
\hline Profundidad & 0,862 & 0,211 & $-0,102$ & 0,855 & 0,553 & $-\mathbf{0 , 8 2 0}$ & \\
\hline $\mathrm{Al}$ & $-0,086$ & 0,426 & 0,237 & 0,073 & $-0,020$ & $-0,024$ & 0,193 \\
\hline
\end{tabular}


TABLA 3

Coeficiente de correlación de Pearson para los metales medidos en el testigo 32A, de la bahía Mejillones. En negritas se indican las correlaciones significativas $(\mathrm{P}<0,001)$

Pearson's correlation table of metals measurement in 32A core, from Mejillones bay. Significant correlations are indicated in bold typing $(\mathrm{P}<0.001)$

\begin{tabular}{lcccc}
\hline & Mo & Cd & $\mathrm{Zn}$ & $\mathrm{V}$ \\
\hline $\mathrm{Cd}$ & 0,476 & & & \\
$\mathrm{Zn}$ & 0,488 & $\mathbf{0 , 7 1 2}$ & & \\
$\mathrm{V}$ & 0,087 & $-0,084$ & $-0,510$ & \\
$\mathrm{Ni}$ & 0,181 & $\mathbf{0 , 7 1 9}$ & $\mathbf{0 , 8 1 8}$ & $-0,417$ \\
\hline
\end{tabular}

En el caso de los metales redox-sensitivos medidos en los sedimentos superficiales, se deben considerar dos factores para explicar acumulación en los sedimentos de fondo de la bahía; el aporte de metales contenidos en el material litogénico (continental) y el flujo biogénico a través de la columna de agua (Nameroff et al. 2002). El primer factor puede ser avaluado mediante el uso del $\mathrm{Al}$ como marcador de fuentes litogénicas (Wehausen \& Brumsack 1998). En el caso de Mejillones, la Fig. 8 muestra que no existe rela- ción entre la concentración de metales traza analizados y la concentración de $\mathrm{Al}$, de manera que se puede interpretar que el aporte de material continental es mínimo y no permite explicar las concentraciones registradas en los sedimentos de fondo. Esta situación es comprensible ya que en la zona de Mejillones el aporte de restos litogénicos a la sedimentación marina está restringido al transporte eólico, el cual representa menos del $5 \%$ del material que sedimenta en la bahía (Vargas 1998).
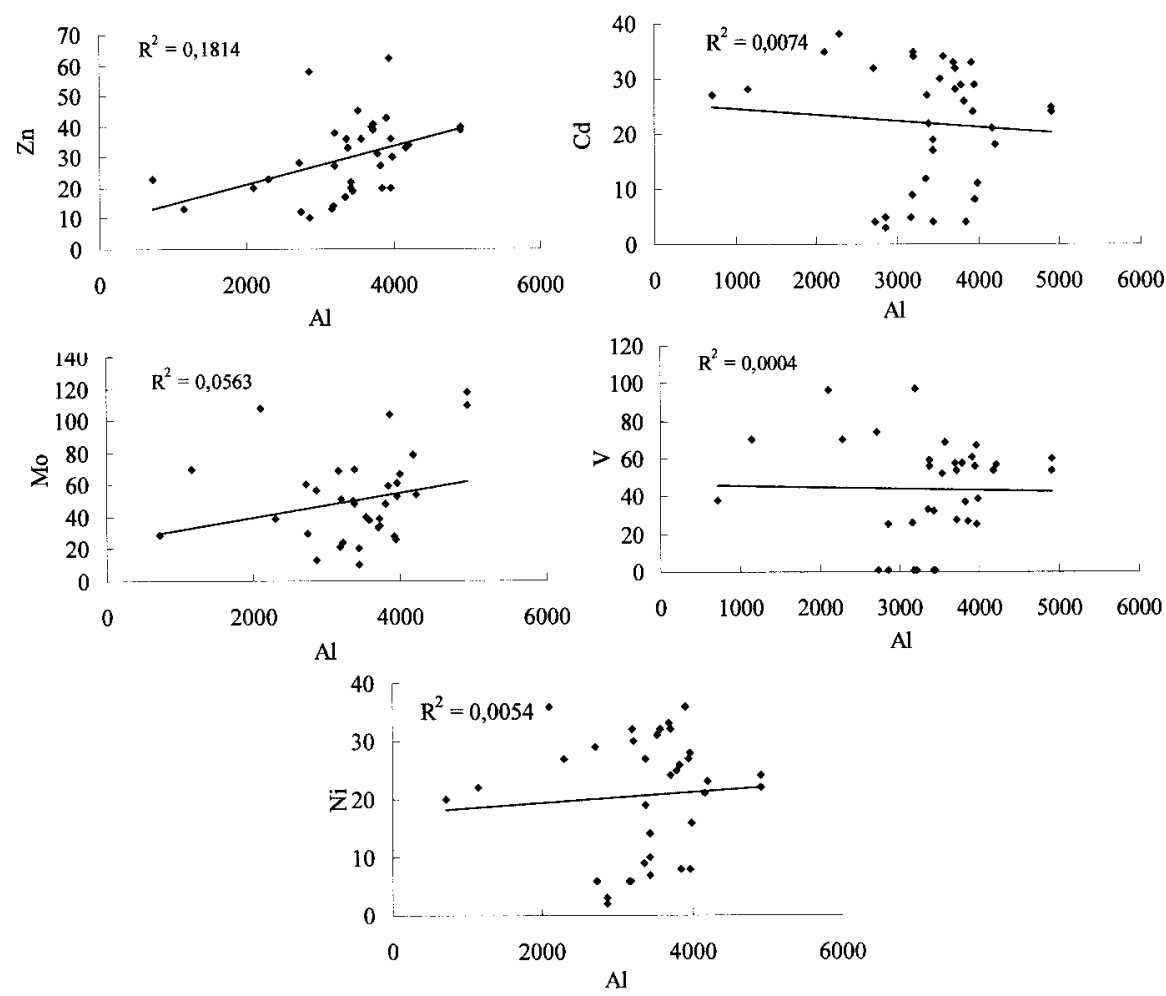

Fig. 8: Correlación Al/metal para las muestras superficiales de la bahía Mejillones.

$\mathrm{Al} / \mathrm{metal}$ correlation for surface samples of Mejillones bay. 
El segundo factor parece ser más importante, debido a que la materia orgánica es el principal constituyente de los sedimentos de fondo de la bahía. Estudios petrográficos y geoquímicas han mostrado que esta materia orgánica es de origen fitoplanctónico (Valdés et al. 2003). Estos organismos tienen incorporado en su estructura una serie de metales, y a medida que sus restos decantan por la columna de agua puede adsorber y transportar hacia los sedimentos de fondo una gran cantidad de metales (Brown et al. 1994). Esto dificulta la evaluación del flujo biogénico debido a que el contenido de metales en el plancton es altamente variable, y depende de la tasa de producción, especies, estructura de la trama trófica, y la concentración de metales disueltos en el ambiente (Nameroff et al. 2002).

Los estudios sobre el contenido de metales en material biogénico tampoco han sido muy numerosos. Los pocos antecedentes que existen muestran que para el caso del $\mathrm{Cd}$, por ejemplo, el contenido presente en los sedimentos de la bahía Mejillones es más alto que el reportado como parte del material biogénico, en la literatura científica. Por ejemplo, Collier \& Edmond (1984) y Kuss \& Kremling (1999) reportan valores de 16,4 y $13,4 \mathrm{mg} \mathrm{kg}^{-1}$ de $\mathrm{Cd}$ en material biogénico, mientras que las muestras superficiales y testigos de Mejillones contienen una media de 21,9 y $37,3 \mathrm{mg} \mathrm{kg}^{-1}$, respectivamente. Esta situación puede indicar que en Mejillones existe otro factor, además del flujo biogénico, que influye en el alto contenido de metales preservado en los sedimentos de fondo.

Por otra parte, la diferencia en el patrón de distribución espacial de todos los metales medidos en Mejillones sugiere que el flujo de partículas no es el único factor que explica el contenido de metales de los sedimentos de fondo. Si este flujo biogénico controlara el aporte de metales al fondo, la distribución de ellos en el sedimentos superficial mostraría un patrón identificable. Los resultados muestran que este no es el caso, ya que solo el $\mathrm{Ni}, \mathrm{Cd}$ y $\mathrm{V}$ presentaron una correlación significativa (Tabla 2). Así, es posible que el flujo biogénico y la acumulación de metales en los sedimentos de fondo sean procesos con cierto grado de independencia. Esto es propuesto por Valdés et al. (2003) para explicar las diferencias encontradas en las tendencias de los perfiles de Cd y carbono orgánico medidos en un testigo. Los autores encontraron que mientras el carbono orgánico disminuyó hacia la superficie, el Cd aumentó, de manera tal que se puede indicar que la materia orgánica no controla, como factor exclusivo, la preservación de este metal en los sedimentos de la bahía Mejillones. Los autores proponen que la disminución del contenido de carbono orgánico en el testigo es una respuesta al decaimiento de la productividad biológica hacia el presente, mientras que la tendencia del $\mathrm{Cd}$ es en respuesta a un reforzamiento de la ZMO hacia la época actual.

Los metales como $\mathrm{Cd}$ y $\mathrm{Ni}$ precipitan siempre en presencia de cantidades traza de $\mathrm{H}_{2} \mathrm{~S}$ (Rosenthal et al. 1995). Este mecanismo de precipitación metal-S puede explicar la alta concentración de metales redox-sensitivos en los sedimentos anóxicos de la bahía Mejillones. Mediciones del Índice de Sulfato Reducción (de acuerdo a Lallier-Verges et al. 1993) realizadas en un testigo recolectado en esta bahía mostraron que la sulfato reducción es un proceso importante en los sedimentos acumulados a $70 \mathrm{~m}$ de profundidad. Así, existen condiciones favorables para la precipitación de complejos metalsulfuro, en el fondo de la bahía Mejillones.

Tomando en cuenta que el oxígeno de los sedimentos proviene de las aguas inmediatamente sobre él, es posible suponer que en Mejillones, a profundidades mayores a $60 \mathrm{~m}$, en donde se han registrado concentraciones de oxígeno disuelto de $0,02 \mathrm{~mL} \mathrm{~L}^{-1}$ (Fig. 2), los sedimentos son totalmente anóxicos. Bajo estas condiciones, los metales redox-sensitivos precipitan y son preservados en los sedimentos aun cuando hayan sido transportados vía flujo biogénico. Estos metales eventualmente pueden difundir hacia la columna de agua, solo cuando las condiciones óxicas afectan a los sedimentos de fondo, disminuyendo la precipitación de metales y dando como resultado la formación de una capa de sedimento caracterizada por un menor contenido de metales redox-sensitivos, del que se formaría durante períodos de condiciones anóxicas de los sedimentos de la bahía Mejillones.

\section{Testigos de sedimento marino}

La estructura estratigráfica predominante en todos los testigos estudiados fue una secuencia de láminas infracentimétricas claras y oscuras, lo cual constituye la primera evidencia de condiciones de depositación caracterizadas por un mínimo de oxígeno (Valdés et al. 2000, Valdés \& Ortlieb 2001, Valdés et al. 2003). Valdés et al. (resultados no publicados) proponen un mecanismo para explicar la formación de láminas claras y oscuras en los sedimentos de la bahía Mejillones. Ellos proponen que una columna de agua bien estratificada, con una oxiclina bien desarrollada y una alta productividad biológica, promueven la formación de láminas oscuras 
constituidas por materia orgánica lábil y refractaria. Por el contrario, una productividad disminuida y, en algunos casos, una columna de agua más oxigenada, dan como resultado la formación de láminas claras, constituida casi exclusivamente por materia orgánica refractaria. Por otro lado, Valdés \& Ortlieb (2001) encontraron que las láminas oscuras presentan un contenido mayor de $\mathrm{Cd}$ y Mo que las láminas claras.

En general, los perfiles de Cd y Mo medidos en tres y dos testigos, respectivamente, son similares. Las pequeñas diferencias registradas pueden ser el resultado de la variabilidad espacial natural, producto de que los testigos fueron recolectados en diferentes puntos de la bahía. Por ejemplo, el testigo 32A fue recuperado en una zona actualmente micróxica, el testigo $32 \mathrm{E}$ fue recuperado en el límite entre la zona micróxica y disóxica, y el testigo $32 \mathrm{G}$ fue recuperado en una zona disóxica (Fig. 2). En este último caso el $\mathrm{Cd}$ y el Mo muestran un notorio decaimiento de la concentración en la parte superior del testigo, lo que podría corresponder a un proceso de remobilización diagenética de estos metales debido a que esta parte de la bahía ha quedado expuesta en el último tiempo a una ambiente de depositación más oxigenado.

\section{Los metales redox-sensitivos como estimadores de} paleoxigenación en la bahía Mejillones del Sur

La concentración de metales en los sedimentos superficiales como función del oxígeno disuelto se muestra en la Fig. 9. Una correlación significativa $(P<0,001)$ e inversa fue encontrada para el $\mathrm{Cd}$ y el $\mathrm{Ni}$, lo que indica que ambos metales aumentan su concentración en los sedimentos cuando el oxígeno disminuye. El análisis de clusters de las muestras de sedimento superficial es más claro al agrupar conjuntamente al Cd, Ni y oxígeno (Fig. 10). Así, ambos procedimientos estadísticos confirman la relación de estos metales con el oxígeno, en la bahía Mejillones. Por otra parte, los mapas de distribución espacial (Fig. 2 y 7) muestran claramente el aumento de la concentración de $\mathrm{Cd}$ y Ni hacia la zona más profunda de la bahía (= menos oxigenada).
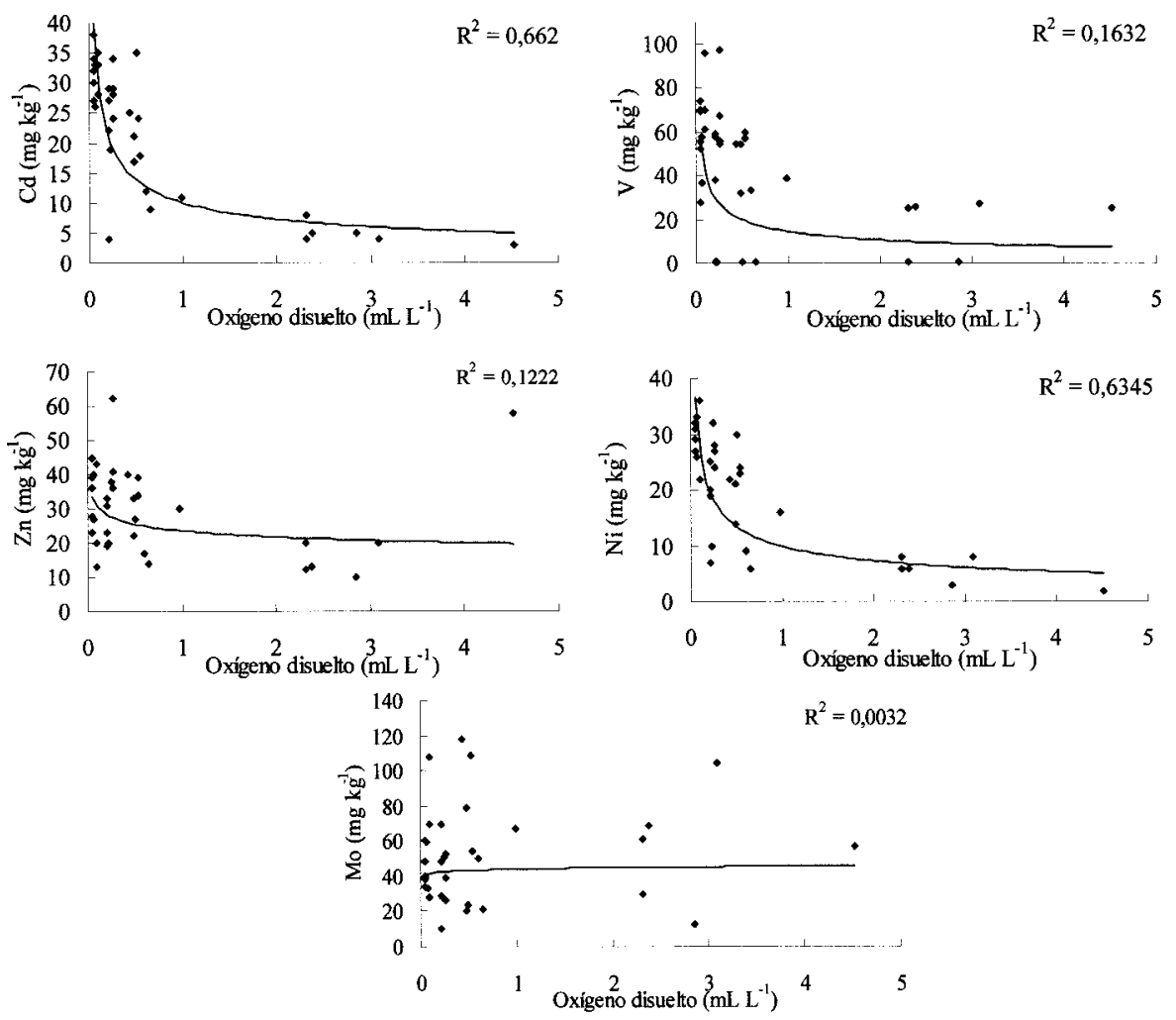

Fig. 9: Correlación entre el oxígeno disuelto de fondo y los metales redox-sensitivos de las muestras de sedimentos superficial de la bahía Mejillones. Se muestra el $\mathrm{R}^{2}$. Concentración de metales en $\mathrm{mg} \mathrm{kg}^{-1}$, y del oxígeno en $\mathrm{mL} \mathrm{L}^{-1}$.

Power correlation between bottoms dissolved oxygen and redox-sensitive metals for surface samples from Mejillones bay. $\mathrm{R}^{2}$ is showed. Metal concentrations in $\mathrm{mg} \mathrm{kg}^{-1}$, and dissolved oxygen in $\mathrm{ml}^{-1}$. 
Nameroff et al. (2002) discuten ampliamente sobre el potencial de diferentes metales redoxsensitivos como estimadores de paleoxigenación. Ellos indican que es difícil su uso para discriminar a un nivel fino, entre condiciones de fondo, óxicas, subóxicas y anóxicas, debido principalmente al efecto del flujo biogénico. Sin embargo, en este trabajo se propone que el registro de $\mathrm{Cd}$ y $\mathrm{Ni}$ en columnas sedimentarias puede ser usado como evidencia de cambios extremos en las condiciones de paleoxigenación, desde anóxico a óxico. Específicamente se propone considerar el fuerte decaimiento en la concentración de $\mathrm{Cd}$ y $\mathrm{Ni}$, dentro de una secuencia sedimentaria caracterizada por altas concentraciones de ambos metales, como una evidencia de episodios óxicos imperantes en el fondo de la bahía, que dieron origen a la formación de láminas sedimentarias con esta composición.

La ZMO del norte de Chile (parte de la ZMO del Pacífico Sureste) está asociada a las Aguas Ecuatoriales Subsuperficiales (AESS) localizadas entre los 50 y $500 \mathrm{~m}$ de profundidad, transportadas por la contracorriente PerúChile (corriente de Günter) (Silva \& Neshiba 1979, Silva \& Sievers 1981, Silva 1983, Arntz et al. 1986). Esta ZMO se encuentra reforzada en las áreas de surgencia activa debido a la degradación de la gran cantidad de materia orgánica que desciende a través de la columna de agua. Bajo esta condiciones, los sedimentos de fondo son típicamente micróxicos o anóxicos, actuando como reservorio o fuente de muchas sustancias, con una importante participación en los ciclos biogeoquímicos.

Datos oceanográficos recolectados durante los últimos años en Mejillones muestran que la posición del límite superior de la ZMO varía a través del año. Esta situación genera la existencia de diferentes ambientes de depositación dentro de la bahía. La Fig. 11 esquematiza la situación media anual para el período 20002001 de la distribución de oxígeno disuelto de las aguas de la bahía. A $35 \mathrm{~m}$ de profundidad los sedimentos son acumulados en un ambiente óxico durante todo el año, mientras que a 75 y $100 \mathrm{~m}$ de profundidad los sedimentos son acumulados bajo condiciones micróxicas/anóxicas, dependiendo de la época del año. Esta situación cambia abruptamente durante períodos El Niño, debido al ingreso de Aguas Subtropicales (AST) que modifican la estructura de la columna de agua, profundizando la oxiclina, y dejando prácticamente a todo el fondo de la bahía expuesta a un ambiente de depositación óxico. Esta situación fue observada, por ejemplo, durante El Niño 1997-1998 (Fig. 12). Mediciones mensuales de oxígeno disuelto realizadas a 80 $\mathrm{m}$ de profundidad, entre junio de 1997 y enero de 1998 (datos proporcionados por Rubén Escribano), mostraron que la ZMO estuvo a una profundidad normal hasta mayo de 1997, y que posteriormente se produjo un brusco aumento del oxígeno de fondo (principalmente en diciembre y enero) asociado a las dos principales ondas Kelvin de El Niño de esa época. Durante ambos períodos el oxígeno alcanzo una concentración de $4,4 \mathrm{~mL} \mathrm{~L}^{-1}$.

La concentración de $\mathrm{Cd}$ en la muestra más reciente de los tres testigos (sedimento superficial) fue de $50,3,40,1 \mathrm{mg} \mathrm{kg}^{-1}$ y $42,0 \mathrm{mg} \mathrm{kg}^{-1}$, mientras que en el mismo sector las muestras superficiales de la segunda campaña mostraron concentraciones de 30,0 y $34 \mathrm{mg} \mathrm{kg}^{-1}$ (Fig. 4).

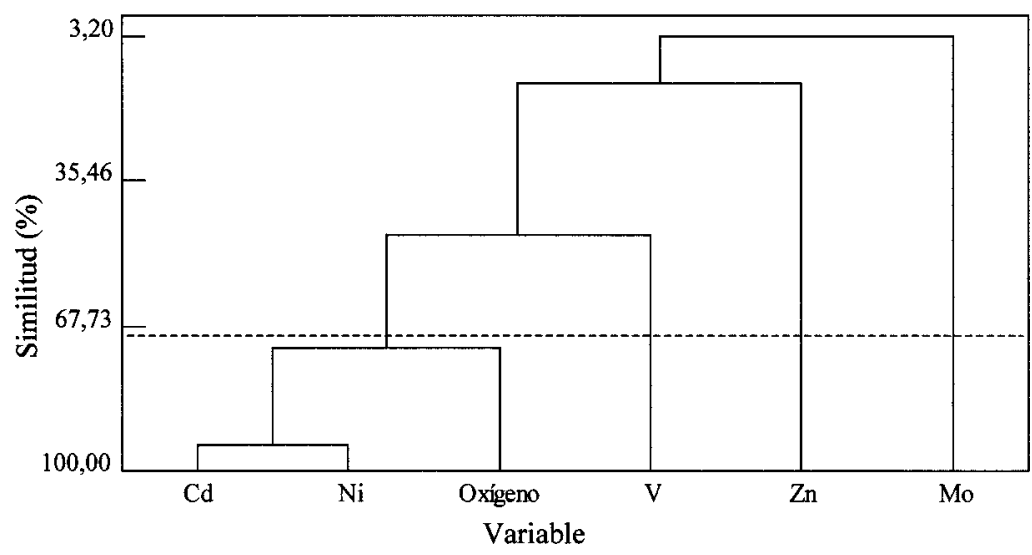

Fig. 10: Análisis de clusters para los metales y oxígeno medidos en la campaña de junio del 2000. Línea punteada corresponde al nivel de similitud utilizado para agrupar los parámetros.

Cluster analysis of metals, oxygen and depth for sampling of June 2000. Dashed line corresponds to similarity level used for to identify different groups. 


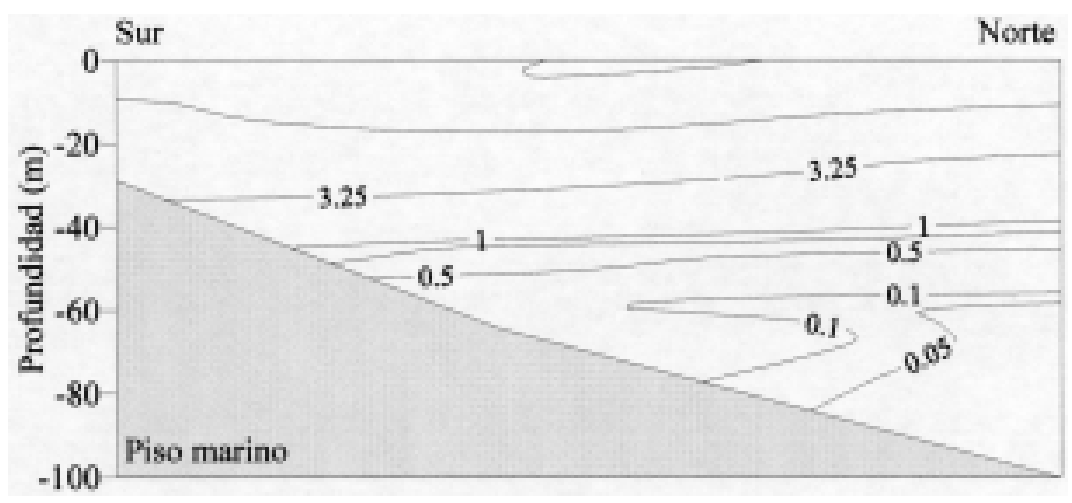

Fig. 11: Sección transversal Sur-Norte ( $\left.70^{\circ} 27^{\prime} 10^{\prime \prime}\right)$ de oxígeno disuelto en la columna de agua de la bahía Mejillones. Concentración de oxígeno en $\mathrm{mL} \mathrm{L}^{-1}$.

South-North $\left(70^{\circ} 27^{\prime} 10^{\prime \prime}\right)$ transversal section of dissolved oxygen in water column from Mejillones bay. Oxygen concentration in $\mathrm{mL} \mathrm{L}^{-1}$.

La concentración de Ni también presentó valores más altos en las muestras más recientes del testigo, que en las muestras de sedimento superficial de la segunda campaña $(45,9$ versus $31.0 \mathrm{mg} \mathrm{kg}^{-1}$ ) (Fig. 7). Esta notoria diferencia es función de las diferentes épocas de muestreo. Las muestras de testigo fueron recolectadas en $1996 \mathrm{y}$, por lo tanto, la parte superior de ellos representa un registro de las condiciones oceanográficas imperante hasta esa fecha. Las muestras de sedimentos superficial $(0,5 \mathrm{~cm})$ fueron recuperadas en el año 2000 y, de acuerdo a tasas de sedimentación para esta zona
(Valdés 1998), representan un registro de las condiciones oceanográficas de los 3-4 años anteriores a esa fecha. Así, se propone que las muestras superficiales de los testigos (especialmente el 32G) representa un registro de condiciones oceanográficas normales (sin El Niño), con sedimentos de fondo anóxicos susceptibles de preservar un alto contenido de $\mathrm{Cd}$ y $\mathrm{Ni}$, mientras que las muestras de sedimentos superficial del año 2000 contienen la señal dejada por El Niño 1997-98, representada por un bajo contenido de $\mathrm{Cd}$ y $\mathrm{Ni}$, debido a un ambiente de fondo más oxigenado.

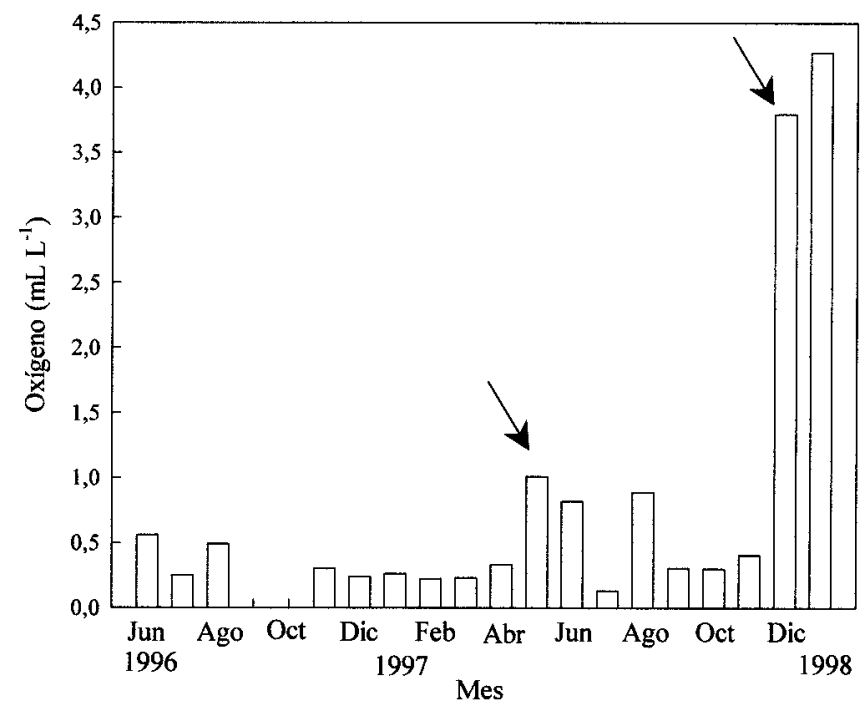

Fig. 12: Variación del oxígeno disuelto de fondo (80 m de profundidad) medidos entre 1997 y 1998. Las flechas indican las principales ondas Kelvin asociadas al evento El Niño.

Bottom dissolved oxygen (80 m depth) in Mejillones bay between 1997 and 1998. Arrows indicate principal Kelvin waves associated to El Niño event. 
El mecanismo propuesto para explicar esta situación se expone en la Fig. 13. Durante períodos no-El Niño ("normales") la estructura de la columna de agua presenta una zona micróxica bien desarrollada, y una interfase agua-sedimentos anóxica. En estos períodos una fracción de los metales disueltos en el agua de mar son transportados hacia el sedimento por el flujo biogénico, mientras que la degradación de la materia orgánica (principalmente en el compartimiento aeróbico de la columna de agua) devuelve parte de estos metales al agua antes de alcanzar el piso marino. Debido a que la degradación de la materia orgánica es mucho menor en ambientes pobres en oxígeno, bajo condiciones no-El Niño, el flujo biogénico es un factor importante que aporta metales desde las aguas superficiales hacia el ambiente subsuperficial. Una vez que la materia orgánica alcanza el piso marino, es eficientemente preservada bajo condiciones anóxicas, y aun cuando parte de ella sea degradada en los sedimentos, los metales redox-sensitivos liberados al agua intersticial y al agua suprayacente al sedimento, rápidamente precipitarán como metal-sulfuro, preservándose en el sedimento. Además, la condición anóxica de la interfase agua-sedimento favorece la precipitación directa de metales como el $\mathrm{Cd}$ y el $\mathrm{Ni}$ desde las formas disueltas en las aguas de fondo. De esta manera se forma un registro sedimentario permanente caracterizado por el alto contenido de $\mathrm{Cd}$ y $\mathrm{Ni}$. Es posible que un registro de las mismas características se forme durante evento La Niña, ya que las condiciones de oxigenación no cambian cuando esta se produce.

Durante períodos El Niño, la situación cambia radicalmente (Fig. 13). La columna de agua y la interfase agua-sedimento permanecen bajo una condición óxica, lo que provoca que una mayor parte de la materia orgánica sea reciclada antes de alcanzar el piso marino, tornando el mecanismo de transporte de metales vía flujo biogénico menos eficiente. Al mismo tiempo, una interfase agua-sedimento más oxigenada favorece la difusión de metales desde la capa superficial de sedimento hacia la columna de agua, y limita el mecanismo de precipitación directa de $\mathrm{Ni}$ y $\mathrm{Cd}$. Así, durante El Niño se forma un registro sedimentario caracterizado por bajo contenido de estos metales, el cual es preservado dentro de la columna sedimentaria, cuando las condiciones oceanográficas vuelven a la normalidad (no-El Niño).

De esta forma, el $\mathrm{Ni}$ y el $\mathrm{Cd}$ pueden constituir una herramienta útil de estudio (desde una perspectiva paleoceanográfica) de variaciones del límite superior de la Zona de Mínimo Oxíge-
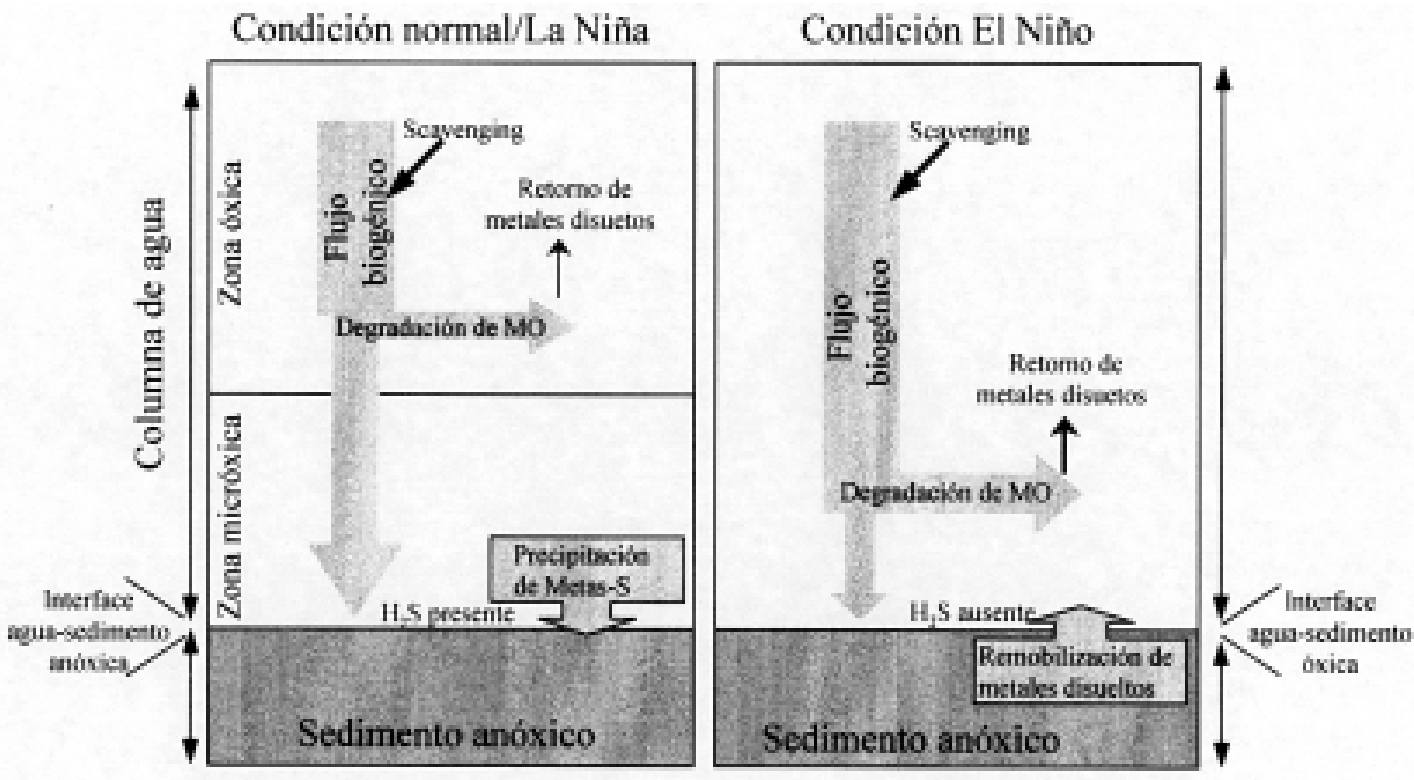

Fig. 13: Esquema de los mecanismos propuestos para explicar la formación de un registro sedimentario correspondiente a periodos El Niño y normales/La Niña, en Mejillones. Para más detalles leer el texto.

Schematic mechanisms proposed to explain the formation of sedimentary record corresponding to non-El Niño/La Niña and El Niño conditions, in Mejillones bay. For further details see the text. 
no, asociadas a eventos El Niño u otros de mayor escala temporal, cuando son registrados en secuencias sedimentarias de la bahía Mejillones.

\section{CONCLUSIONES}

De los cinco metales redox-sensitivos analizados en este trabajo, solo el $\mathrm{Cd}$ y el $\mathrm{Ni}$ parecen responder como estimadores de paleoxigenación en la bahía Mejillones. Ambos metales presentaron una distribución espacial asociada a la variación del oxígeno disuelto de las aguas de fondo. Se propone que estos metales pueden ser usados como estimadores de cambios extremos en la oxigenación, desde un ambiente de fondo micróxico (interfase agua-sedimento anóxico) a uno óxico. Estos cambios abruptos pueden generar secciones dentro de la columna de sedimento con diferentes concentraciones de estos metales. Así, por ejemplo, El Niño 19971998 que cambió la condición normalmente micróxica del ambiente de fondo de la bahía Mejillones a un ambiente óxico, puede explicar las diferencias encontradas en la concentración de $\mathrm{Cd}$ y Ni en la parte más superficial de los testigos (campaña de 1996) y las muestras de sedimento superficial (campaña de año 2000).

\section{AGRADECIMIENTOS}

Este trabajo fue financiado por el proyecto FONDECYT 2960074, y por el proyecto PEI 1370 de la Universidad de Antofagasta. Se agradece al Institut de Recherche pour le Developpement (IRD, Francia) y a la Universidad Central de Venezuela (Caracas, Venezuela) por su apoyo en el análisis de parte de las muestras de sedimento. También se agradece el valioso aporte de los correctores anónimos que ayudaron a mejorar este manuscrito.

\section{LITERATURA CITADA}

ANDERSON R \& J GARDNER (1989) Variability of the late Pleistocene-Early Holocene oxygen-minimum zone off northern California. Geophysical Monograph 55: 75-84.

ARNTZ W, J TARAZONA, V GALLARDO, L FLORES \& H SALZWEDEL (1986) Benthos communities in oxygen deficient shelf and upper slope areas of the Peruvian and Chilean Pacific coast, and changes caused by El Niño. Modern and Ancient Continental Shelf Anoxia, Geological Society Special Publication 58: 131-154.

BERNHARD J \& B SEN-GUPTA (1999) Foraminifera of oxygen-depleted environments. En: Sen-Gupta B (ed) Modern Foraminifera: 201-216. Kluwer Academic Publishers, Cambridge, Great Britain.
BOSTICK B S FENDORF \& M FENDORF (2000) Disulfide disproportionation and CdS formation upon cadmium sorption on $\mathrm{FeS}_{2}$. Geochemica et Cosmochemica Acta 64: 247-255.

BROWN J, A COLLING, D PARK, J PHILLIPS, D ROTHERY \& J WRIGHT (1994) Ocean chemistry and deep-sea sediments. The Open University/Pergamon Editions, Oxford, United Kingdom. 133 pp.

BROWN E, L CALLONEC \& CH GERMAN (2000) Geochemical cycling of redox-sensitive metals in sediments from lake Malawi: a diagnostic paleotracer for episodic changes in mixing depth. Geochimica et Cosmochimica Acta 64: 3515-3523.

CALVERT S, R BUSTIN \& T PEDERSEN (1992) Lack of evidence for enhanced preservation of sedimentary organic matter in the oxygen minimum of the Gulf of California. Geology 20: 757-760.

CALVERT S \& T PEDERSEN (1993) Geochemistry of recent oxic and anoxic marine sediments: implications for the geological records. Marine geology 11: 67-88.

CALVERT S, R BUSTIN \& E INGALL (1996) Influence of water column anoxia and sediment supply on the burial and preservation of organic carbon in marine shales. Geochimica et Cosmochimica Acta 60: 1577-1593.

COLLIER R \& J EDMOND (1984) The trace element geochemistry of marine biogenic particulate matter. Progress in Oceanography 13: 113-199.

CRUSIUS J \& J THOMSON (2000) Comparative behavior of authigenic Re, $\mathrm{U}$, and Mo during reoxidation and subsequent long-term burial in marine sediments. Geochimica et Cosmochimica Acta 64: 2233-2242.

DEAN W, J GARDNER \& D PIPER (1997) Inorganic geochemical indicators of glacial-interglacial changes in productivity and anoxia on the California continental margin. Geochimica et Cosmochimica Acta 61: 4507-4518.

DIDYK B, B SIMONEIT, S BRASSELL \& G EGLINTON (1978) Organic geochemical indicators of paleoenvironmental conditions of sedimentation. Nature 272: 216-222.

ESCRIBANO R (1998) Population dynamics of Calanus chilensis in the Chilean eastern boundary Humboldt Current. Fish. Oceanography 7: 245-251.

ESCRIBANO R \& P HIDALGO (2000) Spatial distribution of copepods in the north of the Humboldt Current region off Chile during coastal upwelling Journal of Marine Biology 80: 1-8.

GONZÁLEZ H, G DANERI, D FIGUEROA, J IRIARTE, $\mathrm{N}$ LEFEVRE, G PIZARRO, R QUIÑONES, M SOBARZO \& A TRONCOSO (1998) Producción primaria y su destino en la trama trófica pelágica y océano profundo e intercambio océano-atmósfera de $\mathrm{CO}_{2}$ en la zona norte de la corriente de Humboldt $\left(23^{\circ} \mathrm{S}\right)$ : posibles efectos del evento El Niño, 1997-98 en Chile. Revista Chilena de Historia Natural 71: 429-458.

GONZÁLEZ H, V ORTIZ \& M SOBARZO (2000) The role of faecal material in the particulate organic carbon flux in the northern Humboldt Current, Chile $\left(23^{\circ} \mathrm{S}\right)$, before an during the 1997-1998 El Niño. Journal of Plankton Research 22: 499-529.

HAMMOND D, J McMANUS, W BERENSON, CH MEREDITH, G KLINKHAMMER \& K COALE (2000) Diagenetic fractionation of $\mathrm{Ge}$ and $\mathrm{Si}$ in reducing sediments: the missing $\mathrm{Ge}$ sink and a possible mechanism to cause glacial/interglacial variations in oceanic Ge/Si. Geochimica et Cosmochimica Acta 64: 2453-2465. 
HELZ G, C MILLER, J CHARNOCK, J MOSSELMANS, R PATRICK, C GARNER \& D VAUGHAN (1996) Mechanism of molybdenum removal from the sea and its concentration in black shales: EXAFS evidence. Geochimica et Cosmochimica Acta 60: 3631-3642.

INGALL E \& R JAHNKE (1994) Evidence for enhanced phosphorus regeneration from marine sediments overlain by oxygen depleted waters. Geochimica et Cosmochimica Acta 58: 2571-2575.

JONES B \& D MANNING (1994) Comparison of geochemical indices used for the interpretation of paleoredox conditions in ancient mudstones. Chemical Geology 111: 111-129.

KAIHO K (1994) Benthic foraminiferal dissolve-oxygen index and dissolved-oxygen levels in the modern ocean. Geology 22: 719-722.

KUSS J \& K KREMLING (1999) Spatial variability of particle associated trace elements in near-surface waters of the North Atlantic $\left(30^{\circ} \mathrm{N} / 60^{\circ} \mathrm{W}\right.$ to $60^{\circ} \mathrm{N} /$ $2^{\circ} \mathrm{W}$ ), derived by large volumes sampling. Marine Chemistry 68: 71-86.

LALLIER-VERGES E, P BERTRAND, A HUC, D BUCKEL \& P TREMBLAY (1993) Control of the preservation of organic matter by productivity and sulphate reduction in Kimmeridgian shales from Dorset (UK). Marine and Petroleum Geology 10: 600-605.

LEGELEUX F, J REYSS, P BONTE \& C ORGANO (1994) Concomitant enrichments of uranium, molybdenum and arsenic in suboxic continental margin sediments. Oceanologica Acta 17: 417-430.

MALCOLM S (1985) Early diagenesis of molybdenum in estuarine sediments. Marine Chemistry 16: 213-225.

MARÍN V, L RODRÍGUEZ, L VALLEJO, J FUENTESECA \& E OYARCE (1993) Efectos de la surgencia costera sobre la productividad primaria primaveral de la Bahía Mejillones del Sur (Antofagasta, Chile). Revista Chilena de Historia Natural 66: 47-491.

MARÍN V \& G OLIVARES (1999) Estacionalidad de la productividad primaria en bahía Mejillones del Sur (Chile): una aproximación proceso-funcional. Revista Chilena de Historia Natural 72: 629-641.

MORFORD J \& S EMERSON (1999) The geochemistry of redox-sensitive trace metals in sediments. Geochimica et Cosmochimica Acta 63: 1735-1750.

MORFORD J, A RUSSELL \& S EMERSON (2001) Trace metal evidence for changes in the redox environment associated with the transition from terrigenous clay to diatomaceous sediment, Saanich Inlet, BC. Marine Geology 174: 355-369.

NAMEROFF T, L BALISTRIERI \& W MURRAY (2002) Suboxic trace metals geochemistry in the eastern tropical North Pacific. Geochimica et Cosmochimica Acta 66: 1139-1158

NAVEA E \& O MIRANDA (1980) Ciclo anual de las condiciones oceanográficas en Mejillones del Sur. Revista de Biología Marina (Chile) 17: 97-133.

ORTLIEB L, O ZÚÑ̃IGA, R FOLLEGATI, R ESCRIBANO, I KONG, L RODRÍGUEZ, PH MOURGUIART, J VALDES \& P IRATCHET (1994) Paleoceanografía de la Bahía de Mejillones del Sur (Antofagasta, Chile): resultados preliminares para el último milenio. Estudios Oceanológicos (Chile) 13: 45-55.

ORTLIEB L, R ESCRIBANO, R FOLLEGATI, O ZÚÑIGA, I KONG, L RODRÍGUEZ, J VALDÉS, N GUZMÁN \& P IRATCHET (2000) Ocean-climatic changes during the last 2,000 years in a hypoxic marine environment of northern Chile $\left(23^{\circ} \mathrm{S}\right)$. Revista Chilena de Historia Natural 73: 221-242.
PÁEZ M, O ZÚÑIGA, J VALDÉS \& L ORTLIEB (2001) Foraminíferos bentónicos recientes en sedimentos micróxicos de la bahía Mejillones del Sur $\left(23^{\circ} \mathrm{S}\right)$, Chile. Revista de Biología Marina y Oceanografía (Chile) 36: 123-133.

PEDERSEN T, M PICKERING, J VOGEL, J SOUTHON \& D ERLE (1988) The response of benthic foraminifera to productivity cycles in the eastern equatorial pacific: faunal and geochemical constraints on glacial bottom water oxygen levels. Paleoceanography 3: 157-168.

RENKA R (1988) Multivariate interpolation of large sets of scattered data. Transactions on Mathematical Software 14: 139-148.

RODRÍGUEZ L, O ZÁRATE \& E OYARCE (1986) Producción primaria del fitoplancton y su relación con la temperatura, oxígeno, nutrientes y salinidad en la bahía de Mejillones del Sur. Revista de Biología Marina (Chile) 22: 75-96.

RODRÍGUEZ L, V MARÍN, M FARÍAS \& E OYARCE (1991) Identification of an upwelling zone by remote sensing and in situ measurement, Mejillones del Sur Bay (Antofagasta-Chile). Scientia Marina 55: 467-473.

ROSENTHAL Y, E BOYLE, L LABEYRIE \& D OPPO (1995) Glacial enrichments of authigenic Cd and U in sub Antarctic sediments: a climatic control on the elements oceanic budget? Paleoceanography 10: 395-413.

RUSSELL A \& J MORFORD (2001) The behavior of redox-sensitive metals across a laminates-massivelaminated transition in Saanich Inlet, British Columbia. Marine Geology 174: 341-354.

SILVA N (1983) Masas de agua y circulación en la región norte de Chile. Latitudes $18^{\circ} \mathrm{S}-32^{\circ} \mathrm{S}$ (Operación oceanográfica MarChile XI- ERFEN II). Ciencia y Tecnología del Mar CONA (Chile) 7: 47-84.

SILVA N \& S NESHYBA (1979) On the southernmost extension of the Peru-Chile Undercurrent. Deep-Sea Research 26A: 1387-1393.

SILVA N \& H SIEVERS (1981) Masas de agua y circulación en la región de la rama costera de la corriente de Humboldt latitudes $18^{\circ}-33^{\circ} \mathrm{S}$. (Operación oceanográfica MarChile X-ERFEN Y). Ciencia y Tecnología del Mar CONA (Chile) 5: 5-50.

SOBARZO M (1994) Oceanografía física entre Punta Nugurne $\left(35^{\circ} 57^{\prime} \mathrm{S} ; 72^{\circ} 47^{\prime} \mathrm{W}\right)$ y Punta Manuel

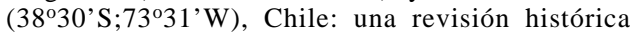
(1936-1990). Gayana Oceanología (Chile) 2: 5-17.

SOBARZO M \& D FIGUEROA (2001) The physical structure of a cold filament in a Chilean upwelling zone (Peninsula de Mejillones, Chile, $23^{\circ} \mathrm{S}$ ). DeepSea Research 48: 2699-2726.

THOMAS A, J BLANCO, M CARR, P STRUB \& J OSSES (2001) Satellite-measured chloroplyll and temperature variability off northern Chile during the 1996-1998 La Niña and El Niño. Journal of Geophysical Research 106: 899-915.

VALDÉS J (1998) Evolución oceanográfica reciente de la Bahía Mejillones del Sur $\left(23^{\circ} \mathrm{S}\right)$. Evidencia geoquímica en sedimentos marinos. Tesis Doctoral, Centro EULA-Chile, Universidad de Concepción, Concepción, Chile. 114 pp.

VALDÉS J \& L ORTLIEB (2001) Paleoxigenación subsuperficial de la columna de agua en la bahía Mejillones del Sur $\left(23^{\circ} \mathrm{S}\right)$ : indicadores geoquímicos en testigos de sedimento marino. Investigaciones Marinas (Chile) 29: 25-35.

VALDÉS J, L LÓPEZ, S LOMÓNACO \& L ORTLIEB (2000) Condiciones paleoambientales de sedimentación y preservación de materia orgánica en Bahía 
Mejillones del Sur (23 S), Chile. Revista de Biología Marina y Oceanografía (Chile) 35: 169-180.

VALDÉS J, L ORTLIEB \& A SIFEDDINE (2003) Variaciones del sistema de surgencia de Punta Angamos $\left(23^{\circ} \mathrm{S}\right)$ y la Zona de Mínimo Oxígeno durante el pasado reciente. Una aproximación desde el registro sedimentario de la bahía Mejillones del Sur. Revista Chilena de Historia Natural 76:347-362.

VAN GEEN A, D McCORKLE \& G KLINKHAMMER (1995) Sensitivity of the phosphate-cadmiumcarbon isotope relation in the ocean to cadmium removal by suboxic sediments. Paleoceanography 10: $159-169$.

VARGAS G (1998) Approches méthodologiques en paléoocéanographie réalisées à partir de carottes de la Baie de Mejillones, Chile (23 $\mathrm{S})$. Diplôme d'Études Approfondies, Université Bordeaux, Bordeaux, France. 32 pp.

VARGAS G (2002) Interactions ocean-atmosphère au cours des derniers siècles sur la côte du Désert d' Atacama: analyse multi-proxies des sédiments laminés de la Baie de Mejillones $\left(23^{\circ} \mathrm{S}\right)$. Tesis Doctoral, Universidad de Bordeaux I, Bordeaux, Francia. $270 \mathrm{pp}$.

WEHAUSEN R \& H BRUMSACK (1998) The formation of Pliocene Mediterranean sapropel: constraints from high-resolution major and minor element studied. En: Robertson A, K Emeis, C Ritcher \& A Camerlenghi (eds) Proceedings ODP, Scientific Results, Leg 160: 201-217. Editorial Texas A\&M University, College Station, Texas, USA.

ZHENG Y, A VAN GEEN, R ANDERSON, J GARDNER \& W DEAN (2000) Intensification of the northeast Pacific oxygen minimum zone during the BoilingAllerod warm period. Paleoceanography 15: 528-536.

ZÚÑ̃IGA O (1974) Cálculo preliminar de la biomasa bentónica de la bahía de Mejillones. Apuntes Oceanológicos (Chile) 6: 1-10.

ZÚÑIGA O, H BAEZA \& R CASTRO (1983) Análisis de la macrofauna bentónica del sublitoral de la bahía de Mejillones del Sur. Estudios Oceanológicos (Chile) 3: 41-62.

Editor Asociado: Matías Medina

Recibido el 26 de mayo de 2003; aceptado el 17 de noviembre de 2003 